\title{
Filigrane
}

Écoutes psychothérapiques

\section{Comment penser la fonction du père ? Vers une vision systémique de la fonction de triangulation}

\section{Raphaële Noël et Francine Cyr}

Volume 19, numéro 1, printemps 2010

Adieu Oedipe, bonjour Narcisse?

URI : https://id.erudit.org/iderudit/044182ar

DOI : https://doi.org/10.7202/044182ar

Aller au sommaire du numéro

Éditeur(s)

Revue Santé mentale au Québec

ISSN

1192-1412 (imprimé)

1911-4656 (numérique)

Découvrir la revue

Citer cet article

Noël, R. \& Cyr, F. (2010). Comment penser la fonction du père ? Vers une vision systémique de la fonction de triangulation. Filigrane, 19(1), 91-106.

https://doi.org/10.7202/044182ar
Résumé de l'article

La conjugalité des parents impose à l'enfant un travail psychique d'aménagement des enjeux d'exclusion et d'inclusion constituant le paradoxe de la triangulation. Nous mettons en évidence un historique des triangulations : celles-ci se définissant comme contenants pour les contenus que sont les fonctions préoedipiennes et oedipiennes du père, ayant elles aussi un historique. Cela nous conduit à plonger dans la mécanique de la triangulation, au plus près de l'interface intrapsychique/interpersonnel : nous développons le concept d'une transitionnalité de la triangulation comme espace de rencontre entre le tiers interne et le tiers externe. La fonction de triangulation classiquement représentée par le père prend alors une dimension systémique : père, mère et enfant contribuent, chacun et ensemble, à l'émergence de cette fonction. La prise en compte de toutes ces considérations nous conduit vers une modernisation du concept d'Oedipe. 


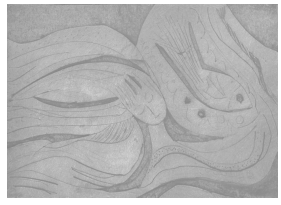

\title{
Comment penser la fonction du père? Vers une vision systémique de la fonction de triangulation
}

\author{
Raphaële Noël et Francine Cyr
}

\begin{abstract}
La conjugalité des parents impose à l'enfant un travail psychique d'aménagement des enjeux d'exclusion et d'inclusion constituant le paradoxe de la triangulation. Nous mettons en évidence un historique des triangulations: celles-ci se définissant comme contenants pour les contenus que sont les fonctions préœdipiennes et œdipiennes du père, ayant elles aussi un historique. Cela nous conduit à plonger dans la mécanique de la triangulation, au plus près de l'interface intrapsychique/interpersonnel: nous développons le concept d'une transitionnalité de la triangulation comme espace de rencontre entre le tiers interne et le tiers externe. La fonction de triangulation classiquement représentée par le père prend alors une dimension systémique: père, mère et enfant contribuent, chacun et ensemble, à l'émergence de cette fonction. La prise en compte de toutes ces considérations nous conduit vers une modernisation du concept d'夭Edipe.
\end{abstract}

\section{Introduction: La rencontre du tiers qui vient de l'intérieur} et du tiers qui vient de l'extérieur

ous nous proposons de reprendre le débat soulevé dans un précédent article (Noël et Cyr, 2009) par la rencontre du père psychanalytique avec le père développemental, afin de pousser plus loin notre réflexion sur la fonction du père et en particulier sur ce qui fait de lui un tiers au quotidien. Le balayage que nous avons fait de la littérature psychanalytique et clinique, et de la littérature développementale et empirique sur la question du père, va servir de cadre à une plongée dans l'univers de la triangulation.

Sous l'influence des recherches interactionnelles sur les relations triangulées père -mère-bébé (Fivaz-Depeursinge et Corboz-Warnery, 2001) et des recherches sur l'interface des univers intrapsychique et interpersonnel à propos des triangulations (Von Klitzing et al., 1995, 1999), nous questionnons la conception freudienne d'un développement séquentiel dans le temps des relations dyadiques et des relations triadiques, au profit de l'hypothèse d'une conception en termes de lignes de développement différenciées. Les compétences triadiques précoces des bébés, ainsi que la présence de schéma d'être à trois dès l'aube de la vie (Stern, 1995, 146) demandent à être intégrées comme d'autres facettes de cette réalité de la triangulation, à côté des 
enjeux qui renvoient au complexe d'Edipe, mais aussi aux triangulations précoces. La rencontre père-enfant se prépare déjà effectivement dans la psyché maternelle, mais aussi dans la psyché paternelle, dimension bien souvent oubliée.

Dans cette perspective, la question de l'origine du tiers reste entière : comment se construit-il ? De l'intrapsychique à l'interpersonnel ou bien dans l'autre sens? Toujours est-il que cette question du passage de la «triadification » (processus interpersonnel qui forme une triade, Von klitzing et al., 1999, 74) à la triangulation œdipienne (processus intrapsychique par lequel la triade est vécue, Von klitzing et al., 1999,74 ) constitue un exemple classique de «l'énigme qui demeure quant aux mécanismes et aux modalités de fonctionnement qui mènent de l'interpersonnel à l'intrapsychique» (Golse, 2002, 883).

Il devient alors nécessaire de faire un pas de côté par rapport à cette logique linéaire pour aller vers une circularité qui nous permette d'envisager des influences mutuelles père-mère-enfant et des co-constructions : conceptions qui prennent leur sens au plan clinique. En effet, lorsque les cliniciens se penchent sur le tiers, ils se demandent par exemple, s'il s'agit d'une «structure toujours-déjà-là » ou bien d'un processus qui prend forme par le biais d'une co-construction au sein de la dyade mère-enfant: "la co-construction d'un espace tiers à vocation paternelle» (Golse, 2006, 157), c'est-à-dire un espace triangulé au sein duquel les différentes fonctions paternelles s'édifient progressivement.

Pour répondre à cette question de l'origine du tiers, il apparaît nécessaire de sonder plus en profondeur la mécanique de la triangulation, ce qui nous conduit du côté d'une métapsychologie de la triangulation. En premier lieu, nous tenterons d'aménager théoriquement ce que nous comprenons de la fonction de triangulation de la conjugalité des parents, dans ce qu'elle suppose d'oscillation entre des enjeux d'exclusion et des enjeux d'inclusion (Vaughn Heineman, 2004, 105).

\section{De la fonction psychique de la conjugalité des parents à la transitionnalité de la triangulation}

La fonction psychique du père au départ conceptualisée par Freud $(1940,12)$ dans le cadre du complexe d'Edipe, semble au fil des différentes théorisations psychanalytiques qui ont suivi, se soutenir de la contribution de chacun des membres de la triade père - mère - enfant (Noël et Cyr, 2009). Le père n'apparaît plus comme le seul responsable d'une fonction de triangulation par ailleurs loin d'être légère à assumer : la mère y apporte sa part par le poids d'une parole désignant le père comme tiers pulsionnellement investi par elle en tant que conjoint et père de son enfant (Fréjaville, 1990, 100; Lacan, 1966, 531), et l'enfant l'utilise psychiquement, par exemple comme troisième espace dans lequel se précipitent toutes les projections négatives relatives au lien mère - enfant, protégeant et réparant ainsi cette relation (dimension développée par les auteurs post-kleiniens dont Bion et Britton, cités par Segal, 1995, 304). Enfin, l'existence chez l'enfant d'une relation à la relation existant entre les parents (Klein, 1932) met en relief la fonction de la conjugalité des parents dans l'aménagement psychique des relations triangulées. 


\section{Le sentiment d'exclusion}

Concernant le vécu de l'enfant face au couple parental, l'accent a tout d'abord été mis sur le sentiment d'exclusion. Ainsi, le fantasme des parents combinés conceptualisé par Melanie Klein (1932) dans le cadre de ce qu'elle a appelé l'Edipe précoce, met en scène un enfant aux prises avec des sentiments d'envie et d'exclusion mais aussi de perte et de privation, sentiments dont la perlaboration le conduira à l'aménagement de la position dépressive. Il imagine ce que les parents font ensemble en dehors de lui et en particulier tout le bon qu'ils peuvent s'échanger, tout ce qu'ils peuvent avoir de nourrissant l'un pour l'autre, sur un spectre allant des enjeux prégénitaux (gratifications orales par exemple) aux enjeux génitaux (couple sexualisé). Il veut à la fois détruire (par la rage de l'envie) et maintenir cette relation. Concernant le sentiment d'exclusion, Emde (1999) en a tracé l'historique développemental pour l'enfant, faisant du sentiment d'exclusion œedipien l'héritier de situations d'exclusion antérieures: exclusion de l'attention des parents, exclusion du contrôle des parents, et enfin exclusion de l'intimité sexuelle des parents.

\section{L'existence concomitante d'enjeux d'exclusion et d'inclusion: le paradoxe de la triangulation}

Cependant, les enjeux dans le triangle œdipien ne se réduisent pas à de l'exclusion. Tout le jeu des rivalités qui se vivent dans ce triangle traduisent la présence de désirs : l'Édipe comme jeu dialectique du désir et du rejet (Perron et Perron-Borelli, $1994,83)$; formulation psychanalytique de la question du paradoxe de la triangulation décrit par Vaughn Heineman $(2004,104)$ comme l'existence concomitante d'enjeux d'exclusion et d'enjeux d'inclusion, à l'intérieur du triangle père-mère-enfant. Il devient alors intéressant de faire un zoom sur ce paradoxe de la triangulation pour tenter de comprendre comment peut se traduire du point de vue l'enfant cette oscillation entre des enjeux d'inclusion et d'exclusion face au couple parental. On peut imaginer le père ou la mère dire au sens large (c'est-à-dire en mots et en attitudes) à leur enfant: tu es exclu de notre intimité sexuelle, expression vivante de notre conjugalité dont tu es issu. L'enfant est issu d'une relation dont il est exclu : voilà l'essence même du paradoxe de la triangulation.

Cette dernière façon de formuler la situation œdipienne élargit le jeu des possibilités, en ajoutant au classique scénario « $2+1$ » (avec toutes les permutations possibles) le scénario « 3 ensemble». Ce qui permet d'intégrer conceptuellement ce qui s'observe au plan relationnel et qui a été bien décrit et documenté par les recherches sur les interactions triangulées père-mère-bébé (Fivaz-Depeursinge et CorbozWarnery, 2001, 41) : la présence de capacités à établir des relations triangulées («triadification » ou triangulation interactionnelles) aussi précocement qu'à quelques mois de vie chez le bébé.

\section{La conjugalité des parents: mécanisme d'action de la triangulation}

Nous proposons que la conjugalité des parents, dans sa façon de rendre incontournable le travail d'aménagement psychique de ce paradoxe (à entendre comme 
paradoxe structurant), constitue le mécanisme d'action de la triangulation, c'est-àdire son principe actif au quotidien. En effet, jour après jour, dans le côtoiement répété de ses parents, l'enfant expérimente les oscillations entre l'exclusion et l'inclusion et travaille à les aménager psychiquement. Ainsi, ce qui se vit dans l'interpersonnel participe à ce qui se construit intrapsychiquement.

Sans aller jusqu'à nous positionner comme Green $(1979,45)$ sur la primauté de l'interprétation et de l'intériorisation de l'expérience sur le vécu de celle-ci, nous convenons qu'il est important de distinguer ces deux registres. Sans les cliver, c'està-dire sans négliger une dimension au profit d'une autre, mais plutôt en concevant l'existence d'une articulation entre l'interpersonnel et l'intrapsychique.

La question de l'énigme du passage de l'un à l'autre (Golse, 2002, 883) peut alors se formuler comme un questionnement sur les conditions d'introjection du tiers, soit « la prise en compte de l'instauration progressive de l'image du tiers dans la psyché de l'enfant» (Golse, 2001, 42). Qu'est-ce qui permet, au plan intrapsychique et au plan interpersonnel, que le tiers puisse s'intégrer psychiquement afin d'avoir une valeur organisatrice dans le fonctionnement psychique? La réponse doit prendre en compte les notions de temporalité en psychanalyse et d'après-coup (Freud, 1918, $53)$ : «le temps où ça se passe n'est pas le temps où ça se signifie. Le temps où ça se signifie est toujours appréhendé rétroactivement» (Green cité par Chaussecourte, 2006, 281). C'est en effet dans la reprise en après-coup (Cournut, 1997, 83), au moyen d'un travail de re-signification des enjeux de triangulation, que peut se mesurer la nouvelle efficacité psychique du tiers (Laplanche, 2005, 129).

\section{Origine du tiers et ontogenèse de la triangulation}

Comment le tiers prend-il une valeur de tiers intrapsychique structurant? Comment est-ce qu'il se re-signifie comme tel? Et plus concrètement, comment le père devient-il un tiers spécifique pour l'enfant?

Tout comme il y a un historique développemental du scénario d'exclusion (Emde, 1999), il y a un historique de la triangulation que nous nous proposons de parcourir afin de bien saisir comment la triangulation œedipienne devient l'héritière des triangulations antérieures. Il y a certes le mécanisme de l'après-coup à considérer pour conférer à la triangulation une efficacité psychique, mais il y a aussi la notion d'un processus qui s'inscrit dans le temps. C'est là une dimension essentielle à côté de laquelle on ne doit pas passer quand on considère la question de l'Edipe, au même titre que pour toutes les étapes du développement psychique de l'enfant (Brusset, 1988). Chaque étape reprenant à son compte les étapes antérieures, dans précisément un travail de re-signification en après-coup. Comme par exemple, l'angoisse de castration reprend à son compte les angoisses de séparation (Cournut, 1997, 33).

Rappelons que les recherches empiriques (Fivaz-Depeursinge et CorbozWarnery, 2001 ; Von Klitzing et al., 1995, 1999) révèlent d'une part, que le bébé est capable très tôt dans la vie, dès les premiers mois, de présenter des interactions triangulées et de participer à des triades. Et qu'elles soulignent d'autre part qu'il existe une interface à penser entre les «triadifications» et les triangulations puisque des 
corrélations sont mises en évidence entre ce que les parents ont aménagé psychiquement en termes de tiers et ce qui se met en scène au plan de l'interaction avec leur bébé. Ceci dit, ce passage de la triade à la triangulation est un «chemin difficile et probablement tortueux» (Golse, 2001, 42).

Au moment où le bébé expérimente des interactions triadiques avec ses parents et des interactions spécifiques avec chacun d'entre eux, contextualisées ou non par l'autre parent, il se construit un univers psychique, au départ étayé sur la relation à l'autre. Ici se définit le domaine de l'intersubjectivité en tant que pont conceptuel entre l'intrapsychique et l'interpersonnel (Lebovici, 2001, 38).

Psychiquement, toutes sortes de situations triangulées sont possibles avec un même parent. Ainsi, Melanie Klein (1943) propose la représentation d'un bébé aux prises avec deux objets partiels relatifs à la mère: le bon sein et le mauvais sein. Ce qui rejoint, dans un registre plus pathologique, la bi-triangulation décrite par Green (1983) dans laquelle l'enfant est en lien avec un même objet parental, mais clivé. Par ailleurs, Green $(1990,263)$ nous rappelle qu'il y a en chaque objet autre chose que luimême et qui n'est pas le sujet: l'autre de l'objet. Aspect double de l'objet qui ici se traduit par le fait que «l'enfant a à faire à un parent qui porte l'autre parent en lui » (Golse, 2001, 43).

C'est donc à l'intérieur même de l'objet mère (au sein de son univers psychique) que le bébé (psychiquement peu différencié de sa mère) fait ses premières rencontres avec le tiers ou plutôt les précurseurs du tiers. Le scénario du bébé en relation avec une mère qui a le père en tête, conceptualisé par Braunschweig et Fain (1975) par la «censure de l'amante » souligne le moment signifiant correspondant au moment où l'enfant devient capable de penser que «si elle n'est pas là, c'est qu'elle est ailleurs » (Diatkine, 1994, 161). Golse $(2006,157)$ va plus loin dans sa façon de définir ces moments où « ce quelque chose du langage de la mère qui échappe à l'enfant délimite un pôle tiers à vocation paternelle ultérieur». Il parle de moments fondateurs d'un espace tiers: "Chaque fois que le bébé peut se dire d'un objet, d'un espace, d'une action ou d'un processus quelconque: « ceci n'est ni-moi-ni-elle», alors il est en train de construire, de forger un espace tiers à vocation paternelle, c'est-à-dire un espace triangulé au sein duquel s'édifieront ensuite les différentes fonctions paternelles proprement dites ».

Bernard Golse $(2006,153)$ nous permet de considérer deux dimensions. Tout d'abord, que cette place de tiers est co-construite par la dyade mère-enfant, qu'elle peut être occupée par des personnages différents et qu'elle deviendra la place du père au sens strict quand celui-ci se signifiera comme tiers particulier. Et ensuite, que c'est dans cet espace triangulé que vont se loger ce que l'on connaît des fonctions du père: fonctions préœdipiennes et œdipiennes que ce même auteur prend soin de décrire comme des processus pouvant avoir lieu dans le même temps, même si les émergences sont séquentielles. En effet le père peut avoir dans le même temps des fonctions de liaison et de protection pour la dyade mère-enfant, ainsi que des fonctions de différenciation et de séparation, ce que nous avons bien exposé dans un autre article (Noël et Cyr, 2009). 
Ainsi, la notion d'historique des triangulations réfère à l'idée que celles-ci s'inscrivent dans le temps et ceci de deux façons. D'une part au plan des contenants, dans un parcours allant vers toujours plus de spécificité: tiercéités partielles, triangulations précoces, place de tiers, place du père. Chaque contenant servant de précurseur pour le suivant. Et d'autre part au plan des contenus, dans le parcours suivant: fonction de liaison, fonction de contextualisation, fonction de différenciation, fonction de séparation. Fonctions dont les émergences s'échelonnent dans le temps, mais dont l'exercice se poursuit de façon concomitante et non pas séquentiellement comme cela fut classiquement établi. D'où l'hypothèse énoncée en début d'article, d'une conception des relations dyadiques et des relations triadiques en termes de lignes de développement différenciées.

En s'inscrivant dans le temps, la triangulation est un processus par lequel l'Édipe peut être conceptualisé comme un moment culminant organisateur sur un continuum d'expériences triangulées (Von Klitzing et al., 1999, 86). L'Edipe apparaît alors comme l'expression la plus aboutie de la triangulation, préparée par toutes les expériences de triangulations précoces. La spécificité de la triangulation œdipienne consiste en un travail de re-signification des triangulations en après-coup chez l'enfant, en prenant en compte la conjugalité des parents comme lien qui l'inclut et qui l'exclut. Cette même conjugalité fait du père un tiers spécifique : l'homme de la mère (le sexuel du lien de couple renvoie à la question de la différence des sexes) et l'homme dont il est le fils ou la fille (la filiation renvoie à la question de la différence des générations).

Cela nous permet de rappeler que le tiers n'est pas seulement un autre différencié. En effet, la triangulation suppose pour l'enfant la reconnaissance et l'intégration de la conjugalité parentale : le tiers est sexué et en lien conjugal avec la mère.

\section{Vers une transitionnalité de la triangulation}

Il nous faut réhabiliter le père de la réalité puisque, tout en ayant une position d'extériorité par rapport à la dyade mère-enfant, il a également une vie psychique peuplée de triangles. Il se prépare à accueillir son enfant, dans toutes les permutations possibles de la triade, en résonance avec la place du tiers en lui et l'histoire de tous ses triangles intériorisés. Il s'agit bien, pour lui comme pour la mère et l'enfant, de faire résonner les expériences d'interactions triangulées (les triades) avec, en lui, les aménagements intrapsychiques du tiers et des triangles.

Il semble que ce soit de cette façon que se construisent les expériences de triadifications (interactions triadiques) et le tiers intrapsychique: expérimenter des triades, dans l'interaction, tout en en ayant des représentations mentales qui soustendent et résultent à la fois des interactions (Golse, 2001, 42). Cela suggère une bidirectionnalité entre l'intrapsychique et l'interpersonnel, une concomitance, une circularité plutôt qu'une linéarité entre le dedans et le dehors. Le sens de construction du tiers est alors à concevoir dans cette idée de bidirectionnalité: dans un allerretour entre l'intrapsychique et l'interpersonnel, avec l'idée déjà énoncée plus haut d'une rencontre du tiers qui vient de l'intérieur avec le tiers qui vient de l'extérieur. 
Le tiers interne permet de donner au tiers externe sa valeur d'agent de triangulation, et l'expérience relationnelle avec le tiers externe vient relancer la construction de la représentation mentale du tiers.

Chez l'enfant, au cours de son développement, la différenciation des espaces internes et des espaces externes est progressive : c'est en se permettant de suspendre la question du dehors et du dedans que cette différenciation est paradoxalement possible. En effet, Winnicott $(1975,26)$ propose avec sa conception d'une aire transitionnelle ou aire d'illusion, une façon d'établir la place de l'objet dans l'entre-deux du dedans et du dehors, du réel et de l'imaginaire, du monde interne et du monde externe. Et c'est paradoxalement la capacité à jouer dans cet espace intermédiaire qui fonde la différenciation entre ces espaces, permettant la création d'un espace psychique interne où viendront se construire et se loger tous les processus de pensée (Chabert, 2007, 558).

De la même façon avec la question du tiers interne et du tiers externe : il faut permettre à l'enfant de jouer avec le tiers, sans avoir à se poser la question du dedans ou du dehors. C'est ainsi que l'on peut penser que se construise un tiers intrapsychique. La transitionnalité appartient au monde psychique, émotionnel et créateur de l'enfant: c'est un mode de fonctionnement psychique spécifique à cette étape de la vie. Il nous faut d'ailleurs y revenir à chaque fois que nous avons à être créatif.

\section{Origine et fonctionnement de l'espace tiers: comment la fonction de triangulation est portée à plusieurs}

Un père qui se transmet via la mère, un père qui se rencontre dans la réalité, un tiers qui émerge de la relation mère-enfant et il faut rajouter: un tiers qui se transmet, via le père. Soulignons le paradoxe qu'il y a dans nos théories explicatives, à faire du père le personnage central de l'Edipe ( «il est la clé de voûte du conflit Edipien universel », J.-L. Le Run, 2004, 127), tout en aménageant si peu de place à son monde intrapsychique, en particulier en ce qui concerne la construction du tiers.

Si le concept de transitionnalité nous permet de concevoir l'émergence du tiers intrapsychique du point de vue de l'enfant ainsi que la rencontre avec le tiers interpersonnel, il nous permet aussi de faire une place à la part d'énigme qui s'y rattache. Nous voudrions maintenant interroger les fondements intrapsychiques de ce tiers, fondements qui sont à l'origine d'une capacité à trianguler. Ce qui consiste à dégager ce qu'il y a de commun et de spécifique dans le psychisme paternel et maternel, pour revenir ensuite au point de vue de l'enfant, toujours dans un souci de va-etvient entre l'intrapsychique et l'interpersonnel.

\section{Construction de l'espace tiers}

Deux considérations s'imposent dans la construction de l'espace tiers. Tout d'abord, père, mère et enfant constituent les pôles d'un triangle renvoyant d'une part à une triade interactionnelle et d'autre part, à un triangle interne construit à partir des représentations mentales de chacun, qu'elles soient en devenir (chez l'enfant) ou en évolution (chez les parents). Ensuite, par delà le vent d'interchangeabilité qu'il y 
a à notre époque concernant les rôles parentaux, il persiste des différences, en particulier des différences sexuées, limitant cette interchangeabilité et produisant des spécificités qui s'avèrent précieuses pour l'enfant du point de vue de son développement (Noël et Cyr, 2009). Ainsi, par exemple, l'existence de la grossesse introduit d'emblée une relation d'intimité entre la mère et l'enfant (Thévenot, 2000, 71) et place le père en position d'extériorité. Il en résulte un travail psychique différent pour la mère et pour le père à certains égards, lorsqu'ils deviennent parents.

Du côté maternel, l'ouverture au tiers se traduit par le fait qu'elle désire ailleurs. Elle ne se satisfait pas exclusivement de son enfant et elle n'a pas l'illusion de pouvoir être toute pour lui. Bien sûr cela suppose qu'elle ait fait le deuil de l'objet parfaitement satisfaisant (ne cherchant ni à l'avoir, ni à l'être, Nasio, 1994) : c'est alors une mère qui reconnaît et accepte l'existence du manque, en soi et chez l'autre. La possibilité qu'il puisse y avoir une place tierce dans son rapport à l'enfant prend alors racine dans cette place reconnue au manque. Julien $(1992,169)$ parle d'une place d'ordre structural, « une place vide, qu'ensuite viendra occuper le père réel ». Ainsi la place du tiers préexiste dans le psychisme de la mère: la métaphore du fauteuil proposée par cet auteur permet de bien nous représenter cette conception d'une place préexistante, dans laquelle viendra se loger le père: «Il faut un fauteuil vide avant de s'y asseoir », écrit-il. Soulignons que cette place tierce que la mère aménage pour le père est teintée d'une part de comment elle investit le père et d'autre part, dans une dimension transgénérationnelle, de ce qu'elle transmet de sa propre histoire infantile: en particulier de son lien avec son propre père (Le Run, 2004, 131) et de ce que sa propre mère a pu lui transmettre de cette place tierce dans son rapport à elle comme enfant.

Ce qui rend difficile ce travail d'aménagement d'une place tierce ou espace tiers (pour élargir le concept) dans son rapport à l'enfant est la nature archaïque de cette relation (archaïque au sens d'indifférenciée). La relation d'intimité favorisée par la grossesse, permet à la mère de se laisser aller à cette "maladie normale» qu'est la préoccupation maternelle primaire (Winnicott, 1958, 285), nécessaire pour décoder les besoins du bébé avec une empathie maximale. En jouant le rôle de contenant pour la dyade mère-enfant, le père permet à la fois que la mère puisse plonger dans cette «folie maternelle primaire» et qu'elle puisse dans un second temps «guérir de cette maladie normale quelques mois après la naissance de l'enfant » (Winnicott, $1958,285)$. Elle en sortira d'autant plus facilement qu'il préexiste en elle une place liée à la reconnaissance du manque, tel qu'évoqué plus haut.

Plusieurs éléments contribuent au fonctionnement de l'espace tiers chez la mère: un travail de différenciation d'avec l'enfant, une conjugalité reconnue et assumée (Julien, 2000, 73), une parole nommant le père comme tiers spécifique pour l'enfant (Golse, 2006, 163) et enfin, une relation suffisamment rassurante et nourrissante offerte à l'enfant, afin qu'elle produise un bon tiers bienveillant Brown, 2002, 276).

Du côté paternel, certaines tâches s'avèrent similaires à celles de la mère, entre autres une capacité de triangulation qui prend également racine dans l'aménagement qu'il fait du manque en lui (deuil de l'objet parfaitement satisfaisant, castration symbolique décrite par Dolto, 1994). Le père a aussi et surtout des tâches différentes 
comme le souligne si joliment le témoignage d'un père (La Gazette, 2007) : "pendant que R. faisait pousser un bébé dans son ventre, moi je faisais pousser un papa dans ma tête ». Il s'agit à la fois de tout le travail de référence à sa propre histoire dans une dimension transgénérationnelle (identification ou contre-identification à son propre père, aux hommes de sa famille) et de tout le travail de redéfinition des repères sociaux qui balisent ce travail psychique de construction de la paternité puisque «être père aujourd'hui, c'est se chercher un modèle»(Cupa, 2000, 98). Enfin, si l'on reprend la métaphore du fauteuil libre (Julien, 1992, 169), le père va occuper à sa façon cette place libre en position de tiers dans le rapport mère-enfant. Il va y mettre ce qu'il est comme homme et ce qu'il transporte comme histoire infantile (Le Run, 2004, 131).

Le fonctionnement de l'espace psychique du père renvoie donc à ce qui le constitue comme tiers. Dans cet ordre d'idée, il a donc un travail psychique bien différent de celui de la mère qui doit s'ouvrir au tiers alors qu'il doit s'employer à incarner le tiers. La position paternelle, marquée par une extériorité première face à la dyade mère-bébé, le pousse à tolérer cette exclusion et à l'élaborer tout en offrant un «holding» à la dyade mère-enfant, favorisant ainsi pour la mère le développement de la préoccupation maternelle primaire (Winnicott, 1958). En même temps, il reste prêt à mettre en action le papa qui a "poussé dans sa tête " pendant la gestation du bébé dans la mère. Ce père se retrouve face à un travail d'investissement (narcissique et objectal) de l'enfant dans un mouvement de rapprochement, inverse au mouvement de différenciation que la mère doit accomplir face à l'enfant. A côté de la construction d'un attachement spécifique à son enfant, il va avoir à exercer des fonctions de séparation et de différenciation pour la dyade mère-enfant, fonctions œedipiennes préparées et potentialisées par l'ensemble des fonctions préœedipiennes (Noël et Cyr, 2009). Ainsi se définit sa fonction de tiers, tout en ayant lui aussi un espace tiers à aménager dans son rapport à l'enfant. Celui-ci se soutient, tout comme pour la mère, d'une conjugalité reconnue et assumée (Julien, 2000, 73).

Du côté de l'enfant, tel que mentionné précédemment, plusieurs travaux mettent en évidence les capacités précoces du bébé à établir des relations triadiques. La construction d'un espace tiers interne se soutient très certainement de ces expériences interpersonnelles, mais aussi de tout le travail d'élaboration psychique des enjeux d'exclusion et d'inclusion vécus au contact du couple parental. L'aménagement de ce paradoxe de la triangulation contribue à la construction du tiers interne et permet à l'enfant d'effectuer un travail de deuil à l'égard d'une position de toute-puissance. L'enfant doit lui aussi aménager le manque : il apprend qu'il n'est pas tout et qu'il ne peut pas tout avoir. Par ailleurs, les auteurs post-kleiniens mettent en relief la capacité qu'a l'enfant d'utiliser son père dans la construction de son appareil psychique, comme par exemple toutes les projections qu'il peut faire sur lui dans l'objectif de réguler le lien à la mère. C'est l'une des façons de conceptualiser la part de l'enfant dans la construction de son espace tiers.

L'espace paternel qui se développe chez l'enfant à partir de l'espace «ni-moi-nielle» que se construit très tôt le bébé, est conçu par Le Run comme « un espace de 
tiercéité, une matrice dans laquelle viendra ou non prendre place le père", une «patrice» $(2004,130)$. Il s'agit là d'une conception de la question paternelle en termes de contenants psychiques dont les contenus correspondraient aux identifications au père, aux représentations imaginaires du père nourries par les différents événements et discours de l'entourage. Cet auteur décrit bien comment la mère et le père contribuent à créer un espace paternel chez leur enfant: la mère contribue autant au contenant (en fondant une place pour le père) qu'au contenu (représentations relatives au père de l'enfant, mais aussi à son propre père : transmission de son propre espace paternel). Il en est de même pour le père dont la tâche spécifique sera d'occuper l'espace paternel de l'enfant en lui proposant des représentations et des identifications (contenus).

Il apparaît alors clairement que cette question du tiers, même si elle est classiquement représentée par le père, est l'affaire de tous et de chacun. Avec une part d'éléments communs, essentiellement organisés autour de l'aménagement du manque en soi. Place conçue comme étant d'ordre structural, c'est-à-dire un contenant pour les contenus en provenance de l'histoire actuelle et passée: les représentations, les identifications et les éléments transgénérationnels issus de l'histoire infantile de chacun des parents par exemple. Puis d'autre part, des éléments spécifiques à chacun : perlaboration du sentiment d'exclusion, aménagement du paradoxe de la triangulation, travail de différenciation, etc. Cet espace tiers, proche de l'espace paternel décrit par Le Run $(2004,129)$, se définit donc aussi en terme de contenant et de contenu.

\section{Vision systémique de la fonction de triangulation}

La fonction de triangulation qui émerge de la construction d'un espace tiers ne doit pas seulement être référée au père: elle apparaît portée par le père, la mère et l'enfant. Chacun y contribue de façon significative, dans l'intimité de son rapport au tiers via la construction de son propre espace tiers. Mais aussi, dans l'expérience des interactions triadiques: le tiers interne et le tiers externe se rencontrant et se construisant mutuellement au sein d'un espace transitionnel symbolisant l'interface interpersonnel/intrapsychique (Von Klitzing, 1999, 74).

Par delà le fait que chacun soit un acteur dans la fonction de triangulation, la mère, forte de son pôle féminin prépare et aménage l'ouverture au tiers, la place du tiers, pour que le père, fort de son pôle masculin, assume l'action d'être ce tiers, incarne le tiers. Quant à l'enfant il nous paraît être à la conjonction de ces deux univers : à la fois s'ouvrant au tiers et allant activement le chercher.

Ces différentes contributions, de la mère, du père et de l'enfant, à la fonction de triangulation s'inscrivent dans la complémentarité et l'interdépendance des rôles de chacun, conformément à une logique circulaire. Nous proposons une vision systémique de la fonction de triangulation dont nous tentons de rendre compte par le schéma de la figure 1. En ce sens nous concevons cette fonction de triangulation comme une propriété émergente du système père-mère-enfant. Le père comme tiers, ou autrement dit: la fonction du père définie comme fonction de triangulation, émerge du travail que chacun effectue relativement à la construction de son espace 
tiers personnel, et des interactions qui ont lieu entre les différents protagonistes dans le champ interpersonnel, interactions soutenues par ce travail intrapsychique et le modelant en retour.

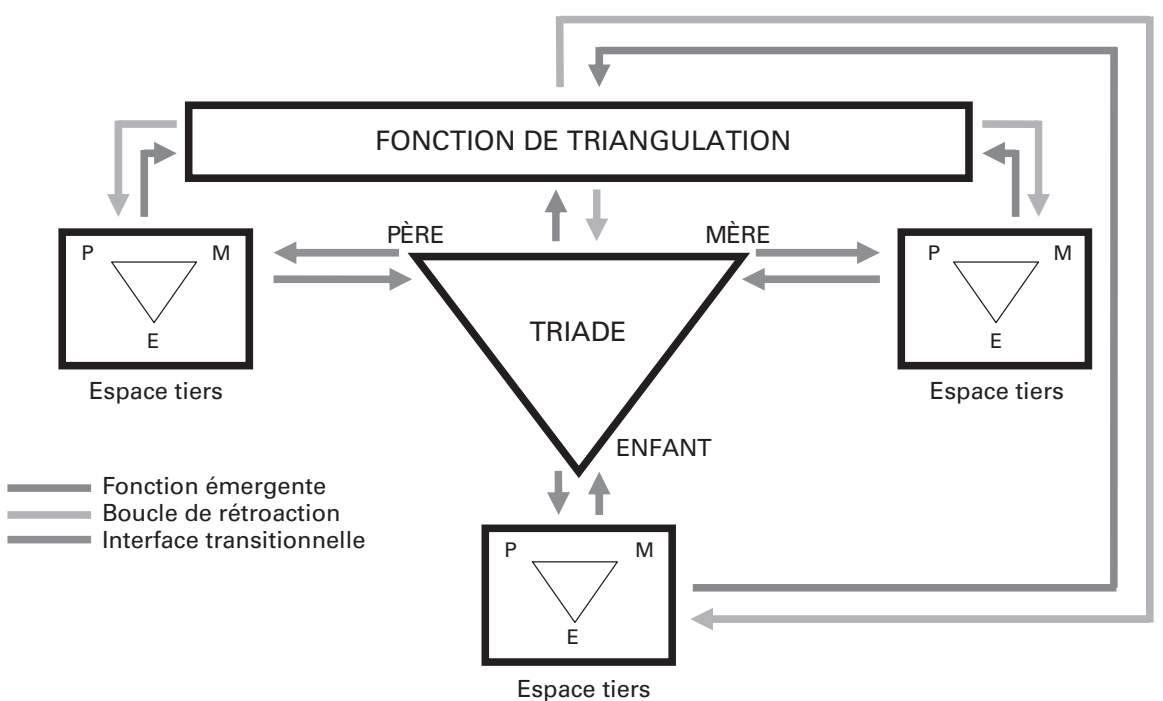

Figure 1. Vision systémique de la fonction de triangulation

La figure 1 illustre que cette fonction de triangulation possède à la fois une dimension spatiale et une dimension temporelle. Les rapports contenant/contenu définissent la dimension spatiale: les triades interpersonnelles (niveau des interactions) servent de contenants aux tiers ou espaces tiers en construction (niveau intrapsychique des représentations mentales et des identifications), contenus modulant et modelant à leur tour les contenants. Par ailleurs, les contenants ont un historique développemental allant des tiercéités partielles à la construction d'une place spécifique pour le père, en passant par les triangulations précoces. Chaque étape sert de précurseur à la suivante pour accueillir les fonctions du père, allant des fonctions de liaisons aux fonctions de séparation, fonctions dont les émergences successives dans le temps constituent l'historique développemental des contenus. La dimension temporelle de la triangulation réside dans tout ce parcours développemental des contenants et des contenus, à l'image des poupées russes, et dans l'idée qu'il s'agit d'une co-construction et d'un co-maintien à trois de cette fonction dans le temps. La spécificité de notre modèle réside entre autres, dans le fait que nous réhabilitons le père comme acteur dans cette co-construction décrite par B. Golse $(2006,153)$ au sein de la dyade mère-enfant.

En effet, la fonction de triangulation psychique met en jeu les trois acteurs du triangle que sont le père, la mère et l'enfant. Nous développons l'idée selon laquelle chacun de ces protagonistes est un pôle actif dans l'actualisation du potentiel de 
triangulation psychique que représente le triangle père-mère-enfant. Chacun a une part de travail psychique à assumer pour soi et pour les autres acteurs, dans la perspective du système.

La métaphore du fauteuil développée par Julien $(1992,169)$ permet de se donner une représentation de l'espace tiers dans son interface intrapsychique/interpersonnelle, au sein du triangle père-mère-enfant. A partir de là, nous proposons d'utiliser cette métaphore pour scénariser des situations traduisant la complexité du phénomène de triangulation, ainsi que les aléas possibles. Ce fauteuil intrapsychique qui fait partie de la mère et qu'elle tend relationnellement au père, peut être présenté par la mère de différentes façons et reçu/occupé par le père de différentes façons:

- Ainsi pour une première situation, un fauteuil est présent chez la mère (un espace tiers) et le père vient s'y asseoir (action du père d'incarner le tiers) : père et mère s'occupent du bébé de façon triangulée, c'est-à-dire en établissant avec l'enfant un rapport qui prend en compte l'autre parent (fonction croisée de la parentalité, Durif-Varembont, 1992, 144).

- Dans un deuxième cas de figure, imaginons qu'il y ait un fauteuil chez la mère mais que le père ne vienne pas s'y asseoir: la mère présente le bébé mais le père ne le prend pas, il n'a peut-être pas fait " pousser un papa dans sa tête».

- Puis une autre situation dans laquelle, il n'y a pas de fauteuil chez la mère (pas d'espace tiers) mais le père va quand même s'asseoir, par terre par exemple (action malgré tout) : il cherche à être présent pour cet enfant en dépit d'une absence d'espace tiers assuré par la mère dans son rapport à l'enfant.

- Enfin, une dernière situation : il n'y a pas de fauteuil chez la mère et le père ne vient pas, ne s'assoit pas: l'enfant reste pris avec sa mère, sans tiers.

Ces différents cas de figure mettent en relief les phénomènes d'alliance ou de collusion qui peuvent avoir lieu entre les parents pour que le tiers existe ou bien soit évacué, dans le rapport à l'enfant. La clinique infantile fait la démonstration régulière que les situations symptomatiques sont beaucoup plus complexes qu'elles n'y paraissent au premier abord et impliquent en général plus d'une personne quand il s'agit de triangulation.

\section{Réhabiliter la part de l'enfant}

Dans le modèle qui sous-tend la métaphore du fauteuil (Julien, 1992, 169), l'enfant n'est pas représenté comme un acteur possible, ce que nous tentons de rétablir avec notre conception systémique de la fonction de triangulation. Au même titre que nous avons cherché à réhabiliter la part du père dans la conception de la coconstruction d'un espace tiers au sein de la dyade mère-enfant (Golse, 2006, 153). La clinique enseigne effectivement qu'il y a des enfants plus ou moins actifs dans leur mouvement vers le père comme tiers. Les difficultés peuvent se situer tant au niveau de la place de tiers co-construite par la dyade mère-enfant, qu'au niveau de la prise de position par le père qui vient s'installer dans cette place en se signifiant comme tiers spécifique (parvient-il ou non à prendre cette position?), mais aussi au niveau de comment le couple négocie cet espace tiers entre eux et pour l'enfant. 
Cette vision systémique nous permet de penser les situations cliniques et familiales, en redonnant à chacun sa part de responsabilité mais au même titre, sa part d'action possible. Ce qui permet de rendre compte de la complexité de la réalité et autorise de ce fait à penser ajuster nos évaluations et nos interventions cliniques auprès des familles. Ainsi :

- Chaque personnage de la triade n'a pas besoin de posséder en soi une pleine capacité à trianguler pour que le système permette l'émergence d'une fonction de triangulation. Il y a des phénomènes de compensation ou de potentialisation qui peuvent avoir lieu entre les parents en termes de forces et de fragilités. Il y a aussi des enfants qui permettent au parent d'être plus facilement un tiers alors que c'est plus ardu avec d'autres parce qu'ils vont plus confronter le parent dans ses points aveugles par exemple, ou bien parce qu'ils ont des besoins particuliers. L'enfant construit sa capacité à trianguler au contact de deux parents arrimant la leur et continuant, on peut le penser, à développer leur propre capacité à trianguler.

- Chaque personnage de la triade possède un potentiel de capacité à trianguler qui va pouvoir s'actualiser dans un triangle père-mère-enfant donné. On peut imaginer qu'un parent ait pu être meilleur ou moins bon tiers dans le contexte d'un couple, plutôt que dans celui d'un autre (cas des recompositions familiales suite à une séparation, par exemple).

Enfin cela nous conduit à l'idée d'une épigénétique de la triangulation : il s'agit pour chaque parent de la rencontre d'un potentiel de triangulation avec un environnement affectif et relationnel, représenté par le cadre de la relation avec l'autre parent, également partenaire amoureux (les dimensions parentale et conjugale s'entrecroisent).

\section{Conclusion: vers une modernisation du complexe d'Сdipe?}

Cette plongée dans l'univers de la triangulation père-mère-enfant nous a permis d'amener notre réflexion plus loin, à la fois dans une dimension intrapsychique, dans une dimension interpersonnelle et dans l'interface de ces deux dimensions. Nos élaborations constituent les bases d'une métapsychologie de la triangulation.

Nous avons fait, d'une part, un pas de plus concernant la fonction symbolique du père vers une définition moins abstraite : la fonction symbolique du père est une fonction de triangulation psychique. L'utilisation de la notion de paradoxe de la triangulation permet d'élargir le spectre des enjeux de la situation oedipienne (enjeux d'exclusion mais aussi d'inclusion) et de rendre compte de la complexité de l'aménagement psychique demandé à l'enfant. A cette occasion, nous soulignons la fonction psychique de la conjugalité des parents dans le travail interne de ce paradoxe.

D'autre part, dans le souci d'articuler ce qui se joue dans l'interaction et dans l'univers intrapsychique en termes de construction du tiers, nous avons eu recours au concept d'aire transitionnelle cher à Winnicott $(1975,27)$. La transitionnalité de la triangulation permet de conceptualiser que c'est dans l'expérience simultanée du tiers interne et du tiers externe que l'espace tiers se construit. 
Un espace tiers qui se fonde également sur tout un travail intrapsychique dont certaines parties sont communes aux parents et d'autres spécifiques au père et à la mère. Sans oublier un enfant à qui l'on redonne une part de responsabilité dans l'actualisation de la fonction de triangulation. Une fonction alors pensée comme une propriété émergente du système père-mère-enfant, ce qui permet de rendre compte des phénomènes d'alliance et de collusion entre les différents acteurs, à l'origine de réalités familiales complexes et de situations cliniques difficiles à démêler.

Enfin, l'image des poupées russes nous permet de rendre compte de la particularité de la dimension temporelle dans laquelle s'inscrit l'émergence de la fonction de triangulation : il y a un historique des interactions triangulées servant de contenant pour les différentes fonctions du père renvoyant à des contenus (représentations mentales, identifications, contre-identifications, discours, événements) qui ont aussi un historique.

La complexité de l'entrecroisement des différents espaces (contenant/contenu) avec une dimension temporelle beaucoup plus complexe que les modalités séquentielles conçues par Freud, nous conduit vers la nécessité d'une modernisation du complexe d'Edipe. Le schéma de Freud est à compléter, enrichi de nos compréhensions et des découvertes des dernières décennies en particulier en ce qui concerne l'articulation du monde intrapsychique et du monde interpersonnel.

Raphaële Noël Clinique spécialisée de pédopsychiatrie Hôpital Rivière-des-Prairies 7070, Boulevard Perras Montréal, Qc, H1E 1A4 raphaele.noel.hrdp@ssss.gouv.qc.ca

Francine Cyr Université de Montréal

\section{Références}

BERGERON, D., 1990, Le féminin, un espace pour le désir, Santé mentale au Québec, XV, 1, 145-164. BRAUNSCHWEIG, D., FAIN, M., 1975, La nuit, le jour. Essai psychanalytique sur le fonctionnement mental, P.U.F., Paris.

BROWN, L. J., 2002, The early oedipal situation: developmental, theoretical, and clinical implications, Psychoanalytic Quarterly, LXXI, 273-300.

BRUSSET, B., 1988, Psychanalyse du lien: La relation d'objet, Le Centurion, Paris.

CHABERT, C., 2007, Les épreuves projectives en psychopathologie de l'adulte, in Roussillon, R., ed., Manuel de psychologie et de psychopathologie clinique générale, Elsevier Masson, Issy-Les-Moulineaux, 551-604.

CHAUSSECOURTE, P., 2006, Observations et psychanalyse. Controverse autour de l'observation des nourrissons, Perspectives Psy, 45, 3, 274-287.

COURNUT, J., 1997, Épître aux Edipiens, P.U.F., Paris.

CUPA, D. et al., 2000, La constellation paternelle pendant la grossesse, in Maury, M., Lamour, M., eds., Alliances autour du bébé. De la recherche à la clinique, P.U.F., Paris, 75-100. 
DIAKINE, R., 1994, L'enfant dans l'adulte ou l'éternelle capacité de rêverie, Delachaux et Niestlé, ParisNeuchâtel.

DOLTO, F., 1994, Les étapes majeures de l'enfance, Gallimard, Paris.

DURIF-VAREMBONT, J.-P., 1992, La fonction croisée de la parentalité, in Clerget, J. et M.-P., ed., Places du père. Violence et paternité, Presses Universitaires de Lyon, 143-153.

EMDE, R. N., 1999, Une progression : les influences intégratrices des processus affectifs sur le développement et en psychanalyse, Revue française de psychanalyse, LXIII, 1, 199-216.

FRÉJAVILLE, A., 1990, Une métaphore polythéiste: la fonction paternelle et ses avatars, Dialogue, 107, 89-102.

FIVAZ-DEPEURSINGE, E., CORBOZ-WARNERY, A., 2001, Le triangle primaire. Le père, la mère et le bébé, Odile Jacob, Paris.

FREUD, S., 1940, Abrégé de psychanalyse, trad, A. Berman, P.U.F, Paris, 1985, 10e éd.

FREUD, S., 1918, À partir de l'histoire d'une névrose infantile, XIII, P., OCF., 1-118.

GOLSE, B., 2006, L'être-bébé, P.U.F., Paris.

GOLSE, B., 2002, Intersubjectif/Intrasubjectif, in Mijolla, A. de, éd., Dictionnaire international de la psychanalyse, Calman-Levy, Paris, 883.

GOLSE, B., 2001, Du corps à la pensée, P.U.F., Paris.

GREEN, A., 1990, De la tiercéité, in Monographie de la Revue française de psychanalyse, P.U.F., Paris, 243-277.

GREEN, A., 1983, La mère morte, in Narcissisme de vie, narcissisme de mort, Minuit, Paris.

GREEN, A., 1979, L'enfant modèle, Nouvelle Revue de psychanalyse, 19, 27-47.

JULIEN, P., 1992, Les trois dimensions de la paternité, in Clerget, J. et M-P., éd., Places du père. Violence et paternité, Presses universitaires de Lyon, Lyon, 167-173.

JULIEN, P., 2000, Tu quitteras ton père et ta mère, Aubier, Paris.

KLEIN, M., 1921-1945, Essais de psychanalyse, Payot, Paris, 1982.

KLEIN, M., 1932, The Psycho-Analysis of Children, The Writings of Melanie Klein, 2, Hogarth, London.

KLEIN, M., 1943, Quelques conclusions théoriques sur la vie émotionnelles des bébés, in Klein, M., Heinman, P., Isaac, S., Rivière, J., eds, Développements de la psychanalyse, P.U.F., Paris, 1968.

LACAN, J., 1966, D’une question préliminaire à tout traitement possible de la psychose, in Écrits, Seuil, Paris, 531-583.

LAPLANCHE, J., 2005, Après-coup, in Mijolla, A. de, éd., Dictionnaire international de la psychanalyse, Calman-Levy, Paris, 128-129.

LEBOVICI, S., 2001, Texte inaugural, in Zaouche-Gaudron, C., ed., La problématique paternelle Érès, Toulouse, 21-48.

LE CAMUS, J., 2001, La fonction du père dans les premières années de la vie de l'enfant. Perspectives ouvertes par la psychologie du développement, in Zaouche-Gaudron, C., éd., La problématique paternelle, Érès, 75-93.

LE CAMUS, J., 2001, La paternité sous les regards croisés de la psychologie du développement et de la psychanalyse, in Zaouche-Gaudron, C., éd., La problématique paternelle, Érès, Toulouse, 145-151.

LE RUN, J.-L., 2004, L'espace paternel à l'adolescence, in Lauru, D., Le Run, J.-L., éds., Figures du père à l'adolescence, Érès, coll. «enfances et psy», Toulouse, 125-138.

NOËL, R., 2008, Du père psychanalytique au père développemental : vision systémique de la fonction de triangulation. Application à l'évaluation de la parentalité psychique, thèse de doctorat en psychologie clinique, Université de Montréal.

NOËL, R., CYR, F., 2009, Le père: entre la parole de la mère et la réalité du lien à l'enfant, La Psychiatrie de l'Enfant, LII, 2, 535-591.

NASIO, J.-D., 1994, Cinq leçons sur la théorie de Jacques Lacan, Petite bibliothèque Payot, Paris.

PERRON, R., PERRON-BORELLI, M., 1994, Le complexe d'Edipe, P.U.F., Que sais-je, Paris, 128.

SEGAL, Hanna, 1995, Le complexe d'CEdipe aujourd'hui, Journal de la psychanalyse de l'enfant, Les psychothérapies psychanalytiques, 22, 297-310.

STERN, D., 1995, La constellation maternelle, Calman-Levy, coll. «Le Passé recomposé », Paris.

THÉVENOT, A., 2000, La structuration de l'enfant et les transformations de la famille: l'exemple des familles recomposées, Le Coq Héron, 161, 70-73. 
VAUGHN HEINEMAN, T., 2004, A boy and two mothers: new variations on an old theme or a new story of triangulation? Beginning thoughts on the psychosexual development of children in nontradional families, Psychoanalytic Psychology, 1, 99-115.

VON KLITZING, K. et al., 1995, Enfant imaginaire, enfant réel et triade, Devenir, 4, 59-75.

VON KLITZING, K. et al., 1999, Child development and early triadic relationships, International Journal of Psycho-Analysis, 80, 71-89.

WINNICOTT, D. W., 1958, La préoccupation maternelle primaire, in De la pédiatrie à la psychanalyse, trad. fr., Payot, Paris, 1969, 285-291.

WINNICOTT, D.W., 1975, Jeu et réalité. L'espace potentiel, Gallimard, «connaissance de l'inconscient», Paris. 